\title{
Study Day of Death Diagnosis
}

National Cancer Institute

\section{Source}

National Cancer Institute. Study Day of Death Diagnosis. NCI Thesaurus. Code C117550.

The study day that a death diagnosis assessment is performed. 